\title{
Progression of Intracranial Meningioma during Luteinizing Hormone-Releasing Hormone Agonist Treatment for Prostate Cancer: Case Report
}

\author{
Takeo ANDA, ${ }^{1}$ Masaru HONDA, ${ }^{1}$ Tokuhiro IsHIHARA, ${ }^{2}$ and Toshiaki KAMEI ${ }^{3}$ \\ ${ }^{1}$ Department of Neurosurgery, Shunan Memorial Hospital, Kudamatsu, Yamaguchi; \\ ${ }^{2}$ Department of Pathology, Tokuyama Medical Association Hospital, Shunan, Yamaguchi; \\ ${ }^{3}$ Department of Pathology, Yamaguchi Grand Medical Center, Hofu, Yamaguchi
}

\begin{abstract}
The authors describe a male patient who developed a large intracranial meningioma during the hormone therapy for pre-existing prostate cancer. A 70-year-old man received a brain check-up, and no intracranial abnormality was detected. Five months later, prostate cancer was diagnosed, and he underwent prostatectomy. Leuprorelin acetate, a luteinizing hormone-releasing hormone (LH-RH) agonist, was subsequently administered to the patient once a month for 3 years. After that he presented with a large parasagittal mass, which was excised. The tumor was histologically diagnosed as meningothelial meningioma, and LH-RH receptors were verified immunohistochemically in the cytoplasm of the tumor cells. Leuprorelin acetate may accelerate the rapid growth of meningioma in this patient.
\end{abstract}

Key words: meningioma, luteinizing hormone-releasing hormone receptor, leuprorelin acetate, prostate cancer

\section{Introduction}

Some sex hormone receptors have been verified in meningioma cells, and several hormones have been reported to affect the growth of meningioma. In this report, the authors present a patient with meningioma whose tumor growth could have been related to a luteinizing hormonereleasing hormone (LH-RH) agonist administration.

\section{Case Report}

\section{History}

A 70-year-old man underwent gastrectomy for early stage gastric cancer, and remained well after that for 7 years. He received a brain check-up at another hospital in November 2007, and no intracranial abnormalities were detected (Fig. 1). In April 2008, early stage prostate cancer (T1C, N0, M0) was detected. Prostatectomy was performed, and leuprorelin acetate and bicalutamide were administered prophylactically to the patient. Monthly treatment was then maintained. In December 2010, he suffered from headache and a brain magnetic resonance imaging (MRI) at another hospital demonstrated an intrac-

Received December 6, 2012; Accepted February 6, 2013 ranial mass lesion. He was referred to our hospital on January 31, 2011.

\section{Examination}

No neurological deficits were observed. Brain MRI revealed a large extraaxial parasagittal mass near the bregma which compressed bilateral frontal lobes and extended into the falx and skull. It showed iso-intense on $\mathrm{T}_{1}$-weighted images (WI) and high-intense on $\mathrm{T}_{2} \mathrm{WI}$, and was homogeneously enhanced after intravenous injection of contrast materials. The diameter of the tumor reached $5 \mathrm{~cm}$ (Fig. 2). Hyperostosis of the skull near the mass was identified by skull X-ray. Many feeding arteries originating from bilateral pial arteries and branches of external carotid arteries were recognized on cerebral angiogram. The superior sagittal sinus (SSS) was segmentally occluded near the bregma. Prostate-specific antigen (PSA) was 0 $\mathrm{ng} / \mathrm{ml}$, carcinoembryonic antigen (CEA) was $1.8 \mathrm{ng} / \mathrm{ml}$, and Ca19-9 was 9.7 U/ml. The tumor was regarded as a parasagittal meningioma invading the falx, SSS, and skull. An operation was performed on March 14, 2011.

\section{Operation}

Under general anesthesia, the medial frontoparietal bone was removed in a divided manner. At the same time, a part of the tumor which invaded the skull was also removed. 

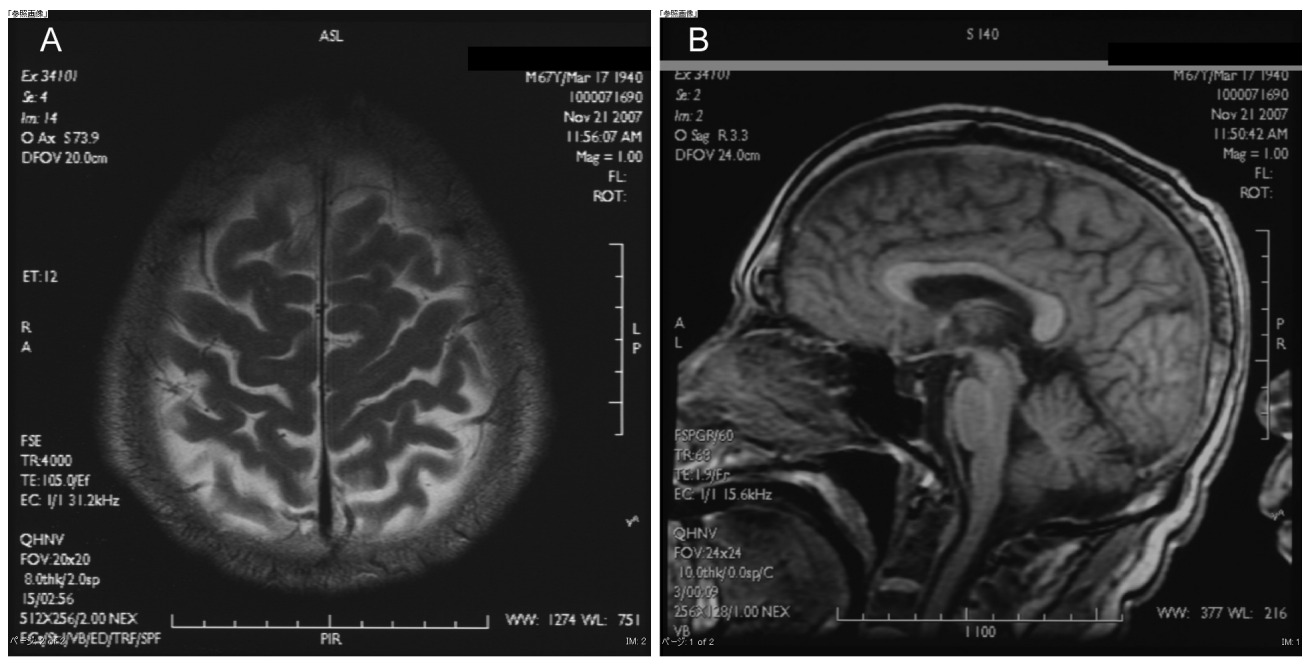

Fig. 1 A: $T_{2}$-weighted axial magnetic resonance (MR) imaging demonstrating no apparent intracranial abnormalities. B: MR sagittal scout imaging demonstrating no apparent abnormalities.
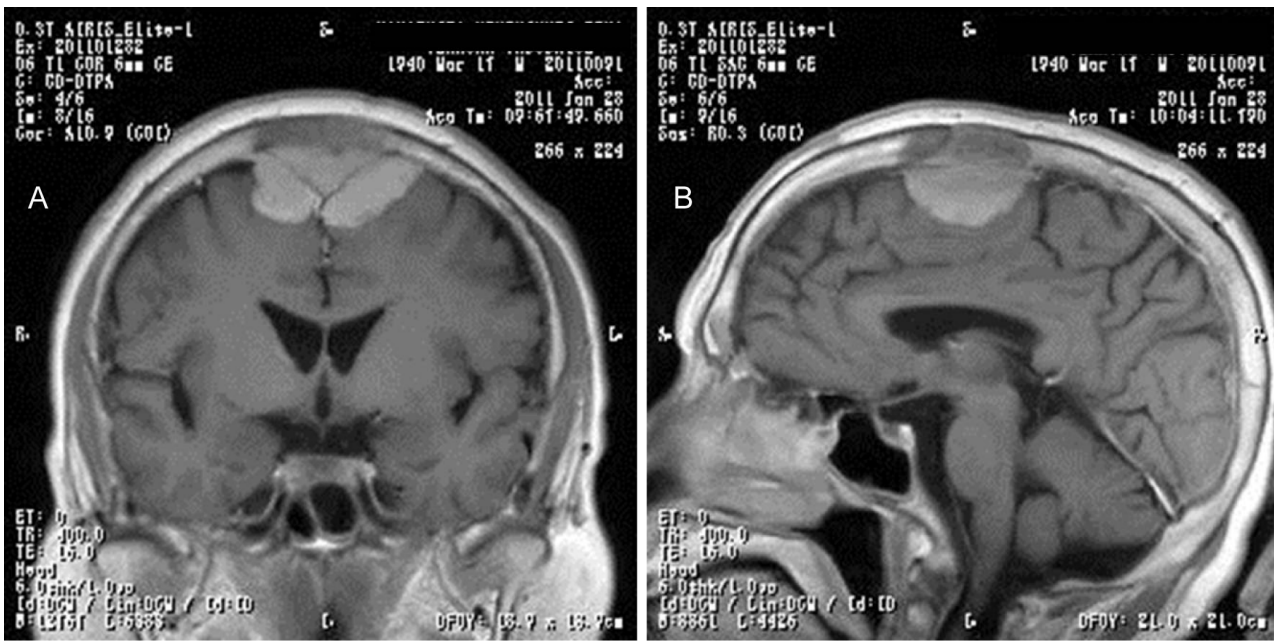

Fig. 2 A: Gadolinium-enhanced $\mathrm{T}_{1}$-weighted coronal magnetic resonance imaging (MRI) demonstrating an extramedullary homogenously enhanced tumor at the parasagittal region expanding into the falx and skull bone. B: Gadolinium-enhanced $T_{1}$-weighted sagittal MRI.

Then, the dura around the tumor was cut, and the border between the tumor and its dural attachment was separated, and the tumor surface was dissected from the brain while interrupting the pial supply. The tumor was pinkish and fragile and tended to bleed. Internal decompression was suitably done, and the tumor was removed in a piecemeal manner. Almost all the tumor attachment, affected falx, and SSS were removed. A part of the attachment near the intact cortical bridging vein was left as it was coagulated. The extent of tumor removal was judged as Simpson grade 2. Dural plasty using Goatex, and cranioplasty using a titanium plate were performed. The tumor was histopathologically proved to be a meningothelial meningioma without atypical or anaplastic features. The molecular immunology borstel-1 (MIB-1) staining index was $5 \%$ or less. Cytoplasmic immunoreactivity for the luteinizing hormone-releasing hormone (LH-RH) receptor was detected (Fig. 3). The used primary antibody was mouse monoclonal anti-LH-RH receptor antibody (Thermo, Fremont, California, USA).

\section{Postoperative course}

There were no perioperative events, and no neurological deficits were recognized. Administration of leuprorelin acetate was ceased. On July 11, 2012, sixteen months after the operation, brain MRI demonstrated no tumor recurrence (Fig. 4).

\section{Discussion}

This patient's intracranial tumor was histologically diagnosed as meningothelial meningioma and it showed no evidence of malignant features. It is very rare for a small benign meningioma not detected by MRI to grow into a 
large mass $5 \mathrm{~cm}$ in diameter, and invade the falx and SSS and skull in the course of less than 3 years. Because bony and dural sinus invasion by meningioma is reportedly not related to its biological malignancy, ${ }^{1-3)}$ the rapid tumor progression in such a short time in this patient is not consistent with typical benign meningiomas. The authors suspect that maintenance of hormone treatment for prostate cancer led to rapid growth of the meningioma.

There are many reports referring to sex steroid hormone receptors in meningiomas. Meningioma occurs in females twice as frequently as in males. Progesterone receptors were found in $83 \%$ of meningiomas, ${ }^{4)}$ and discontinuation of a progesterone agonist induced regression of meningioma. ${ }^{5)}$ Antiprogestational therapy showed efficacy for unresectable meningioma. ${ }^{6}$

Receptors of gonadotropin-releasing hormone, namely LH-RH were identified immunohistochemically in the cytoplasm of $95.1 \%$ of meningiomas resected. ${ }^{7)}$ Another report demonstrated that LH-RH increased proliferation of meningioma cells in vitro. ${ }^{8)}$

Leuprorelin acetate, an LH-RH agonist, exhibits antiandrogenic effect if administered to males, so it is often used for patients with prostate cancer. The authors found a few reports referring to leuprorelin acetate administered and a meningioma developing in patients with prostate cancer. Fallanca et al. reported two cases. A 70-year-old man had undergone hormonal therapy with leuprorelin (7.5 mg/month) and bicalutamide $(150 \mathrm{mg} /$ day $)$ for 3 years after radical prostatectomy and radiotherapy for prostate cancer. Subsequently, abnormal tracer uptake in the lumboaortic lymph nodes and lumbosacral vertebral body and the posterior cranial fossa were found by a (11C) choline positron emission tomography/computed tomography (PET/CT) scan. The lesions in the skeletal bone and lymph nodes were thought to be metastasis, but the latest was highly suggestive of meningioma on enhanced MRI. Hormonal treatment was discontinued and chemotherapy with docetaxel was started. After two cycles of chemotherapy, PET/CT showed that the intensity of abnormal tracer uptake in the metastatic lesion was increasing but was stable in the posterior cranial fossa meningioma. ${ }^{9)}$ This article quoted other two cases described in a case report written by Lee and Terris. ${ }^{10)}$ Both patients developed neurological symptoms during LH-RH agonist treatment after surgery for prostate cancer. In one patient, meningioma was found on radiological imaging, and in the other, who had undergone surgery for meningioma, tumor recurrence was documented.

Bicalutamide is an androgen receptor antagonist, and is often administered to patients with prostate cancer. An effect of this drug on the development of meningioma is regarded as unlikely.

As mentioned above, leuprorelin acetate, an LH-RH agonist, could conceivably play a role in the rapid growth of meningioma. This hypothesis is consistent with the immunoreactivity for the LR-RH receptor in the tumor cells of this patient. Nevertheless, there have been only
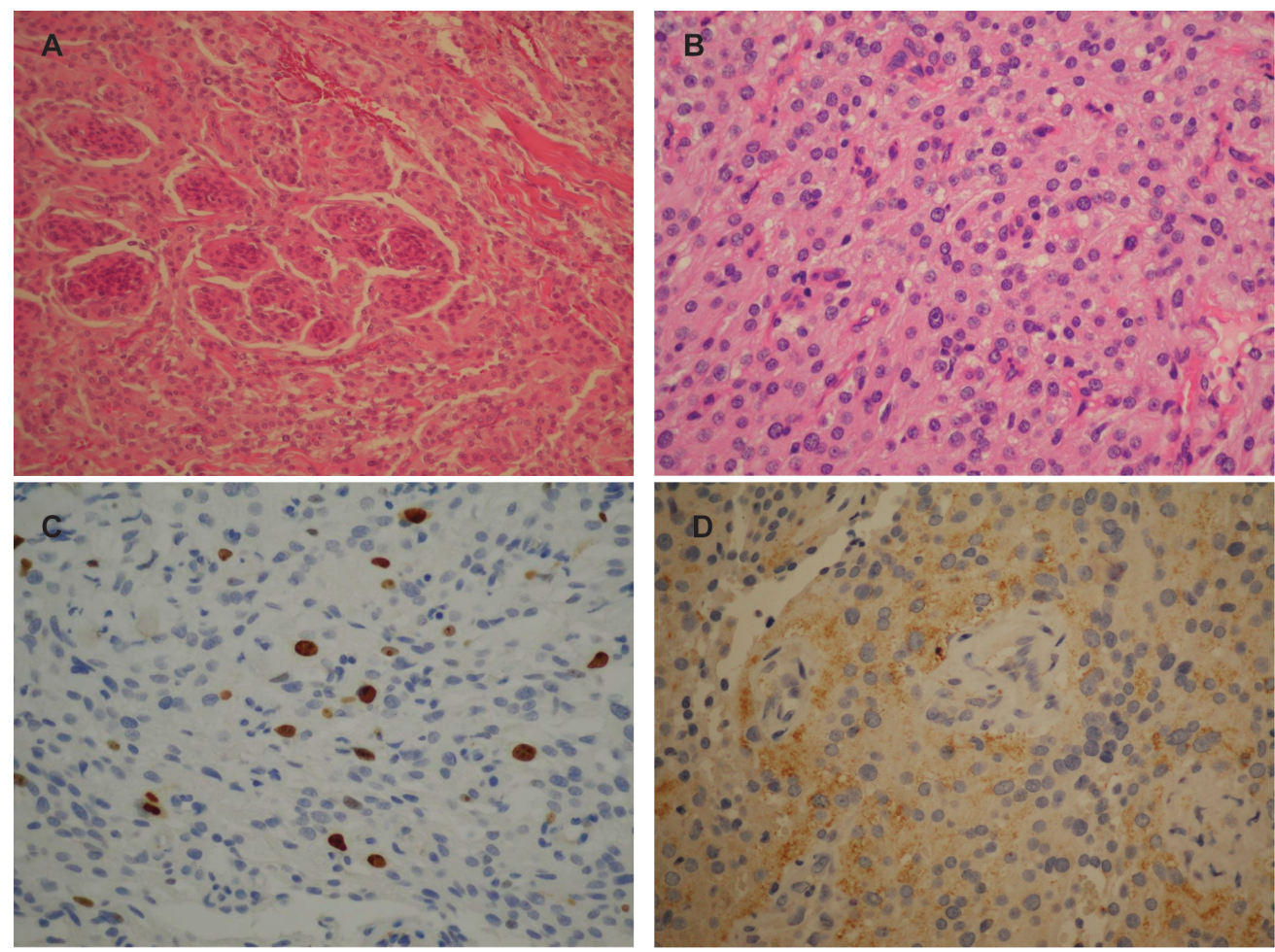

Fig. 3 Photomicrographs of the resected tumor. A: Whorl arrangements are seen in places. Necrosis is not identified. Hematoxylin and eosin (H\&E) staining, original magnification $\times \mathbf{2 0 0}$. B: Tumor cells are largely uniform and have oval nuclei. Mitoses are not identified. H\&E staining, original magnification $\times 400$. C: Molecular immunology borstel (MIB)-1 staining index is $\mathbf{5} \%$. Immunohistochemical stain for Ki-67, original magnification $\times 400$. D: The cytoplasm of tumor cells is immunopositive for the LH-RH receptor. Immunohistochemical stain for the LH-RH receptor, original magnification $\times 400$. LH-RH: luteinizing hormone-releasing hormone. 

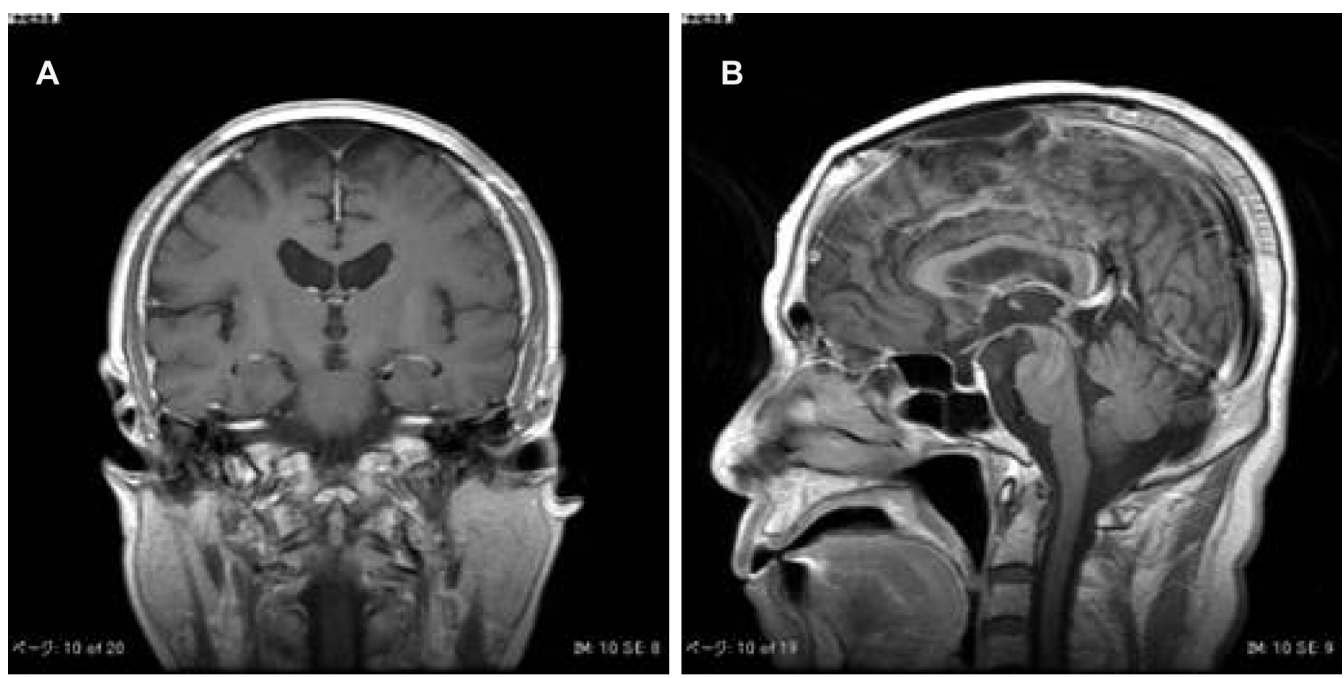

Fig. 4 No recurrent tumor is identified 16 months after the operation. A: Gadoliniumenhanced $\mathrm{T}_{1}$-weighted coronal magnetic resonance imaging (MRI). B: Gadolinium-enhanced $\mathrm{T}_{1}$-weighted sagittal MRI.

a few reports of this kind. This may be because there may be very few male patients with prostate cancer who have meningioma simultaneously as it predominantly occurs in females. Also, PSA, a strong tumor marker for prostate cancer at present, was not popular before. So, some patients with prostate cancer in early stage might be overlooked. Further clinical experience and investigation are needed.

\section{Conclusion}

The authors documented a 70-year-old man with prostate cancer whose meningioma had grown to $5 \mathrm{~cm}$ in diameter over 3 years during hormone treatment for prostate cancer. In this patient, leuprorelin acetate, an LH-RH agonist, could have contributed to the rapid growth of meningioma.

\section{Acknowledgements}

The authors thank the laboratory technician, Ms. Tomoko Hayaki, for her assistance in the immunohistochemistory of the tumor specimen.

\section{Conflicts of Interest Disclosure}

None. Two authors who are the members of Japan Neurosurgical Society have already submitted the conflict of interest self-declaration to the society.

\section{References}

1) DiMeco F, Li KW, Casali C, Ciceri E, Giombini S, Filippini G, Broggi G, Solero CL: Meningiomas invading the superior sagittal sinus: surgical experience in 108 cases. Neurosurgery 55: 1263-1272; discussion 1272-1274, 2004
2) Moon HS, Jung S, Jung TY, Cao VT, Moon KS, Kim IY: Possible role of matrix metalloproteinase in osteolytic intracranial meningiomas. J Korean Neurosurg Soc 47: 11-16, 2010

3) Raza SM, Gallia GL, Brem H, Weingart JD, Long DM, Olivi A: Perioperative and long-term outcomes from the management of parasagittal meningiomas invading the superior sagittal sinus. Neurosurgery 67: 885-893; discussion 893, 2010

4) Hsu DW, Efird JT, Hedley-Whyte ET: Progesterone and estrogen receptors in meningiomas: prognostic considerations. J Neurosurg 86: 113-120, 1997

5) Shimizu J, Matsumoto M, Yamazaki E, Yasue M: Spontaneous regression of an asymptomatic meningioma associated with discontinuation of progesterone agonist administration. Neurol Med Chir (Tokyo) 48: 227-230, 2008

6) Grunberg SM: Role of antiprogestational therapy for meningiomas. Hum Reprod 9(Suppl 1): 202-207, 1994

7) Hirota Y, Tachibana O, Uchiyama N, Hayashi Y, Nakada M, Kita D, Watanabe T, Higashi R, Hamada J, Hayashi Y: Gonadotropin-releasing hormone (GnRH) and its receptor in human meningiomas. Clin Neurol Neurosurg 111: 127-133, 2009

8) Durmaz R, Deliorman S, Isiksoy S, Uyar R, Tel E: Luteinizing hormone releasing hormone increases proliferation of meningioma cells in vitro. Archives Physiol Biochem 107: 286-291, 1999

9) Fallanca F, Giovacchini G, Picchio M, Bettinardi V, Messa C, Fazio F: Incidental detection by [11C]choline PET/CT of meningiomas in prostate cancer patients. Q J Nucl Med Mol Imaging 53: 417-421, 2009

10) Lee KL, Terris MK: Luteinizing hormone-releasing hormone agonists and meningioma: a treatment dilemma. Urology 62: 351,2003
Address reprint requests to: Takeo Anda, MD, Department of Neurosurgery, Shunan Memorial Hospital, 1-10-1 Ikunoyaminami, Kudamatsu, Yamaguchi 744-0033, Japan. e-mail: tanda-s@carol.ocn.ne.jp 